\title{
Acetaminophen changes paracellular transport activity through regulation of the tight junction protein in an intestinal barrier model
}

\author{
Mohammad R Lornejad-Schäfer ${ }^{1,2^{*}}$, Christine Schäfer ${ }^{1,2}$, Klaus R Schröder $^{1,2}$ \\ From 18th Scientific Symposium of the Austrian Pharmacological Society (APHAR). Joint meeting with the \\ Croatian, Serbian and Slovenian Pharmacological Societies. \\ Graz, Austria. 20-21 September 2012
}

\section{Background}

Drugs and diet interact with each other in mutually influencing their bioavailability. In our previous work we have shown that $N$-acetyl- $p$-aminophenol (APAP, acetaminophen) reduces the paracellular transport activities of itself and co-administered substances. The aim of this study was to find out how APAP reduces the bioavailability of small molecules which pass paracellularly, and which tight junction proteins are involved in the regulation of paracellular transport using a Caco-2 barrier model.

\section{Methods}

The Caco-2 in vitro model is widely used by the pharmaceutical industry to predict the absorption of orally administered drugs. To construct a Caco-2 barrier model, the Caco-2 cell line, a continuous line of heterogeneous human epithelial colorectal adenocarcinoma cells, was seeded onto inserts (Millipore, $0.4 \mu \mathrm{m}$ pore size) for 21 days. After differentiation, the cells were treated topically with $10 \mathrm{mM}$ APAP for $24 \mathrm{~h}$. The cell transepithelial electrical resistance (TER) and capacitance $(\mathrm{Ccl})$ were determined by two different impedance-measuring systems. The membrane permeability was tested by differently sized molecules: Lucifer Yellow (520 Da), 3-5 kDa and $40 \mathrm{kDa}$ Fluorescein thiocarbamoyl (FITC)-dextran. The tight junction proteins (ZO-1, occludin) were investigated using Western blot analysis and immunofluorescence staining.

\section{Results}

APAP increased the TER value, i.e. membrane integrity, which correlated significantly with the decrease of

\footnotetext{
* Correspondence: lornejad@zet.or.at

'BioMed-zet Life Science GmbH, zet-LSL, 4020 Linz, Austria

Full list of author information is available at the end of the article
}

permeability of the small molecules Lucifer Yellow (ca. $520 \mathrm{Da})$ and FITC-dextran (3-5 kDa) but not of large molecules (40 kDa FITC-dextran). The tight junction protein $\mathrm{ZO}-1$ and its tyrosine phosphorylation were upregulated after $24 \mathrm{~h}$ treatment with APAP.

\section{Conclusions}

Tight junction proteins play an important role in maintaining the intestinal barrier function. We assume that APAP affects the paracellular transport pathway through disassembly of the tight junction. We suppose that APAP leads to remodelling of the tight junction protein $\mathrm{ZO}-1$ through changes in the level of protein expression and in tyrosine phosphorylation. APAP may reduce the bioavailability of co-administered substances through remodelling the tight junction by regulating $\mathrm{ZO}-1$ and its tyrosine phosphorylation. As a result, the paracellular transport activity for small molecules is decreased as is net intestinal absorption.

\section{Acknowledgements \\ This work was supported by the Province of Upper Austria. \\ Author details \\ ${ }^{1}$ BioMed-zet Life Science GmbH, zet-LSL, 4020 Linz, Austria. ${ }^{2}$ zet-Centre for Alternative and Complementary Methods to Animal Testing, 4020 Linz, Austria.}

Published: 17 September 2012 